\title{
The Relationship Between Health-Related Quality of Life and Saliva C-Reactive Protein and Diurnal Cortisol Rhythm in Latina Breast Cancer Survivors and Their Informal Caregivers: A Pilot Study
}

Thaddeus W.W. Pace*, ${ }^{1-4}$ Associate Professor of Nursing, Psychiatry, and Psychology, twwpace@email.arizona.edu

Terry A. Badger, ${ }^{1,2,4}$ Professor of Nursing and Psychiatry, tbadger@email.arizona.edu

Chris Segrin, ${ }^{5}$ Professor of Communication, segrin@email.arizona.edu

Alla Sikorskii, ${ }^{1,6}$ Professor of Psychiatry, and Statistics and Probability, Alla.Sikorskii@hc.msu.edu

Tracy E. Crane, ${ }^{4,7,8,9}$ Assistant Professor of Nursing, Public Health, and Nutritional Sciences tecrane@email.arizona.edu

${ }^{1}$ Division of Community and Systems Health Science, College of Nursing, University of Arizona, Tucson, AZ, United States; ${ }^{2}$ Department of Psychiatry, College of Medicine, University of Arizona, Tucson, AZ, United States; ${ }^{3}$ Department of Psychology, College of Science, University of Arizona, Tucson, AZ, United States; ${ }^{4}$ University of Arizona Cancer Center, Tucson, AZ, United States, ${ }^{5}$ Department of Communication, College of Social and Behavioral Sciences, University of Arizona, Tucson, AZ, United States; ${ }^{6}$ Department of Psychiatry, College of Osteopathic Medicine, Michigan State University, East Lansing, MI, United States; ${ }^{7}$ Division of Biobehavioral Healthscience, College of Nursing, University of Arizona, Tucson, AZ, United States; ${ }^{8} \mathrm{Mel}$ and Enid Zuckerman College of Public Health, University of Arizona, Tucson, AZ, United States; ${ }^{9}$ Department of Nutrition Science, College of Agriculture and Life Sciences, University of Arizona, Tucson, AZ, United States.

Funding: V Foundation for Cancer Research (D2015-009, PI: T.A. Badger), American Cancer Society (RSG-12-120-01-CPPB, PI: T.A. Badger)

*Corresponding Author and contact for Public and Scientific Queries: Thaddeus Pace, PhD, Associate Professor, College of Nursing, University of Arizona, 1305 N Martin Ave, Tucson, AZ 85722, United States; +1 520-626-3520 (office), +1 520-626-7891 (fax), twwpace@email.arizona.edu 


\begin{abstract}
INTRODUCTION: To date no studies have explored associations between objective stressrelated biomarkers (i.e., inflammatory markers, diurnal rhythm of cortisol) and health-related quality of life (HRQOL) in Latina breast cancer survivors and their informal caregivers (i.e., family, friends).
\end{abstract}

METHODS: This cross-sectional feasibility study assessed saliva C-reactive protein (CRP), saliva diurnal cortisol rhythm (cortisol slope), and self-reported HRQOL (psychological, physical, social domains) in 22 Latina survivor-caregiver dyads. Feasibility was defined as $\geq 85 \%$ samples collected over 2 days (upon waking, in afternoon, and in evening). Associations between biomarkers and HRQOL were examined with correlational analyses.

RESULTS: Collection of saliva was feasible. Strongest associations were observed between survivor evening cortisol (as well as cortisol slope) and fatigue, a component of physical HRQOL.

DISCUSSION: Associations presented may help promote investigations of mechanisms linking stress-related biomarkers and HRQOL in Latina breast cancer survivor-caregiver dyads, which will facilitate development of culturally congruent interventions for this underserved group. 
Latinas in the United States experience poorer health-related quality of life (HRQOL) both during and after treatment for breast cancer than non-Hispanic whites (NHWs) (Luckett et al., 2011; Yanez, McGinty, Buitrago, Ramirez, \& Penedo, 2016; Yanez, Thompson, \& Stanton, 2011). HRQOL is a multidimensional construct that includes psychological, physical, social, and spiritual domains (Ferrell et al., 1996), and Latina breast cancer survivors have been found to have poorer psychological (i.e., reduced general mental health, increased distress, greater depressive symptoms), physical (increased fatigue and pain), and social HRQOL compared to NHWs, Blacks, and Asian Americans (Luckett et al., 2011; Yanez et al., 2011).

HRQOL impairments associated with breast cancer are not just experienced by breast cancer survivors, but also by their informal caregivers (family or friends), who provide supportive care both during and after treatment. Both suffer from psychological HRQOL impairments (depressive symptoms and distress) around the time of diagnosis, as well as during and after primary treatment for cancer have ended (Cubukcu, 2018; Kent et al., 2016; Lambert, Girgis, Lecathelinais, \& Stacey, 2013; Munoz et al., 2017). HRQOL impairments experienced by informal caregivers are important for the well-being of breast cancer survivors, because HRQOL in breast cancer survivors has been found to be interdependent with HRQOL in informal caregivers (Segrin \& Badger, 2014). That is, impairments in a given domain of HRQOL among caregivers at one point in time may predict impairments in the same domain in breast cancer survivors at a later point, or vice versa.

With respect to the possible mechanisms involved, available evidence suggests that disproportionate HRQOL impairments for Latina breast cancer survivors and their caregivers persist even after controlling for socioeconomic status, cancer diagnosis, and cancer treatments 
(Carver, Lehman, \& Antoni, 2003; Eversley et al., 2005; Spencer et al., 1999). HRQOL experienced by Latina survivors and their caregivers must therefore involve other factors.

Stress-based theories of health suggest that stressors related to ethnic minority status may contribute to HRQOL impairments experienced by Latina breast cancer survivors (Yanez et al., 2011), perhaps by way of stress-related biological pathways (i.e., inflammation and cortisol). In support of this hypothesis is research with breast cancer survivors of different ethnicities that has found associations between HRQOL impairments (i.e., depressive symptoms, affect, and fatigue) and circulating concentrations of inflammatory biomarkers (i.e., various proinflammatory cytokines, or the proteins that immune cells use to communicate with one another, as well as Creactive protein $[\mathrm{CRP}])($ Bower, 2019; Han et al., 2016; Xiao et al., 2017) as well as flattening of the normal diurnal cortisol rhythm (i.e., less fluctuation from higher circulating or saliva concentration in the morning, to lower concentrations in the evening)(Bower et al., 2005; GieseDavis, Wilhelm, et al., 2006). Diurnal cortisol rhythm has been associated with other dimensions of HRQOL in survivors, including perceived stress (Juarez Garcia, Landero Hernandez, González-Ramírez, \& Jaime, 2016), social support or perceived social support (Ho, Fong, Chan, \& Chan, 2013; Turner-Cobb, Sephton, Koopman, Blake-Mortimer, \& Spiegel, 2000), and emotional expression (Giese-Davis, DiMiceli, Sephton, \& Spiegel, 2006). Distress has also been associated with biomarkers of inflammation and diurnal cortisol rhythm in caregivers (Miller et al., 2014), and alternations of these markers have been found when comparing caregivers to controls (Park, Ross, Klagholz, \& Bevans, 2018). However, the degree to which HRQOL is associated with inflammation and diurnal cortisol rhythm in Latina breast cancer survivors or their caregivers has not been explored, nor has the value of stress-related biomarkers for research on improving delivery of culturally congruent health care for these survivors and caregivers. 
Including objective assessments of stress-related biomarkers in research with culturallytailored interventions designed to promote wellness for Latina breast cancer survivors and their informal caregivers could help to improve effectiveness of such interventions, by "fine tuning" intervention elements related to psychosocial stress. Latina survivors and their caregivers may be more likely to be exposed to everyday psychosocial stressors than non-Hispanics, including stressors that are commonly encountered by those who are members of minority groups in the United States (e.g., discrimination) (Yanez et al., 2011). Including objective biomarkers in research with Latina survivors and caregivers may also eventually improve interpretation of selfreport assessments of HRQOL in research intended to develop culturally-tailored interventions, so that self-reports may better reflect the internal states of Latina survivors and their caregivers. Finally, evidence suggests that there may be mistrust in Latinos regarding the collection and storage of samples for biomarkers, as well as fear of harm and worries about collection burden (G. Moreno et al., 2015). Research is therefore needed to demonstrate acceptability of these markers for Latina survivors and their caregivers before transcultural nursing research with biomarkers and wellness in these survivors and caregivers can proceed.

The goals of the present study were to 1) establish feasibility to collect saliva samples from Latina breast cancer survivors and their informal caregivers for determination of diurnal cortisol rhythm and concentrations of CRP, an inflammatory biomarker, and 2) explore how activity of stress-related biological pathways may associate with HRQOL in the same group. Saliva samples were collected by participants in the home setting. Of note, concentrations of cortisol and CRP have been found to associate with concentrations of the same biomarkers in the circulation (i.e., plasma or serum)(Kirschbaum \& Hellhammer, 1994; Ouellet-Morin, Danese, Williams, \& Arseneault, 2011). Although saliva cortisol has been assessed in breast cancer 
survivors before, to the best of our knowledge saliva CRP has not. And both biomarkers have never been assessed in Latina survivor-caregiver dyads. We predicted it would be feasible to collect saliva for analyses of these stress-related biomarkers in Latina breast cancer survivors and their informal caregivers.

\section{MATERIALS AND METHODS}

\section{Sample and Study Design}

Twenty-two dyads consisting of Latina breast cancer survivors and their informal caregivers (i.e. family member or close friends [named by the survivor]) from southern Arizona took part in this cross-sectional study between 2013 and 2017. Participants were a subset of a larger sample that was recruited for a parent study that tested the effects of two different 8-week telephone-based psychosocial interventions to improve HRQOL for Latina breast cancer survivors and their informal caregivers [author citation]. The current study was conducted at baseline prior to the initiation of any intervention. HRQOL assessments part of the parent study were reviewed by an advisory board $(\mathrm{AB})$ consisting of 5 Latino representatives who were either breast cancer survivors or informal caregivers. Changes were made to HRQOL assessments based on $\mathrm{AB}$ comments, to make the assessments more acceptable to the study population . The $\mathrm{AB}$ also reviewed and approved biomarker procedures for the present study. Although the $\mathrm{AB}$ endorsed the plan to collect saliva, they did not endorse the collection of blood from survivors and caregivers. The study was a preliminary effort, and was not designed to formally test hypotheses about associations between biomarkers and HRQOL. Instead, the intention was to determine estimates of effect sizes. Formal hypothesis-based sample size determination was therefore not applicable. However, we selected a target sample size of at least 12 dyads, and enrolled additional dyads from the parent study when available to account for possible attrition, 
based on guidelines suggesting that a group of this size would be sufficient to determine feasibility of collecting saliva for assessment of stress-related biomarkers (Julious, 2005). All participants of the pilot study provided written informed consent for procedures of the parent and pilot study, including saliva sampling. Procedures were approved by [university name removed] Human Subjects Protection Program and [university name removed] Institutional Review Board.

\section{General Procedures and Collection of Saliva Samples}

After recruitment and consent, survivors and caregivers were provided saliva collection kits to be used starting within 24 hours of assessment of HRQOL domains. HRQOL assessments were completed by telephone interview. Participants then collected their own saliva in the home setting using Salivette saliva collection tubes (Sarstedt, Nümbrect, Germany) over two consecutive days at three time points (immediately upon awakening in the morning, between 4:30 and 6:00 PM, and bedtime) (Sephton et al., 2009; Spiegel, Giese-Davis, J., Taylor, C.B., \& Kraemer, H., 2006). Saliva collection kits included a detailed, one-page long instruction sheet in either Spanish or English (depending on the participant's preferred language) that clearly instructed participants about when to collect saliva over the 2-day period and what behaviors to avoid when collecting saliva (e.g., within 30 minutes of consumption of anything except water, or brushing teeth). The instruction sheet provided a section for participants to write down the time when each saliva sample was collected. Participants were contacted by telephone and/or by text message with reminders to begin saliva collection. Participants were instructed to place saliva samples in their home freezer immediately after collection and until pickup by study staff for transport back to the [name of laboratory removed per instructions for authors] on dry ice. Survivor demographic and cancer treatment information were obtained by accessing participant medical records. Informal caregiver height and weight were collected by self-report. 


\section{Cortisol and C-reactive protein}

Saliva samples were analyzed for cortisol in duplicate using a competitive enzyme immunoassay (EIA) kit (Salimetrics, State College, PA) following manufacturer instructions. The assay used $25 \mu \mathrm{l}$ of saliva in duplicate per determination and had a lower limit of sensitivity of $0.006 \mu \mathrm{g} / \mathrm{dl}$. Intra and inter-assay coefficients of variation of the cortisol EIA were $8.4 \%$ and 9.1\%, respectively. Saliva samples were assayed for concentrations of CRP in duplicate using an enzyme linked immunosorbent assay (ELISA) kit (Salimetrics, State College, PA), following manufacturer instructions and as previously used by Danese and colleagues (Ouellet-Morin et al., 2011). The assay used $50 \mu 1$ of sample diluted $10 \mathrm{X}$ before determination in duplicate and has a lower limit of detection of $46.88 \mathrm{pg} / \mathrm{ml}$. Intra and inter-assay coefficients of variation for the CRP ELISA were 3.7\% and 4.9\%, respectively. Personnel who performed laboratory procedures to determine saliva concentrations of biomarkers in the laboratory did not have access to HRQOL assessment data or other participant information collected for the study.

\section{Health-related Quality of Life Assessments}

Instruments used to assess HRQOL were administered in each participant's preferred language (Spanish or English) by a bilingual data collector over the telephone, at a time convenient for the participant. HRQOL was divided into psychological (depression, anxiety, negative affect, perceived stress), physical (fatigue), and social (social isolation, family functioning, emotional support, informational support) domains (Ferrell et al., 1996). We used PROMIS scales to assess many of these domains (Cella et al., 2010). There were 5 response options for each item on PROMIS scales $(1=$ never, 5 = always $)$, with higher total scores indicating greater amounts of the HRQOL domain measured (Cella et al., 2010). Raw total scores were transformed into t-scores, for which the general population has mean 50 and 
standard deviation 10. Personnel who administered HRQOL assessments did not have access to biomarker data collected by the study. Psychological HRQOL. Symptoms of depression were measured with the PROMIS Distress-Depression short form 8a, and Centers for Epidemiological Studies Depression Scale (response options: $0=$ rarely or none of the time, $3=$ most or all of the time; score range from 0 to 60 with higher scores indicting greater depression symptoms)(Radloff, 1977). For the PROMIS Distress-Depression the internal consistency reliability was .94 among survivors, and .93 among caregivers. For the CESD, the internal consistency reliability was .95 among survivors, and .89 among caregivers. Symptoms of anxiety were measured with the PROMIS Emotional Distress -Anxiety short form 8a. The internal consistency reliability was .94 among survivors and .92 among caregivers. Both positive affect and negative affect were measured with respective subscales of the Positive and Negative Affect Schedule (response options: 1 = very slightly or not at all, 5 = extremely; scores range from 10 to 50 on each subscale), with higher scores reflecting higher levels of affect in either domain (Watson, Clark, \& Tellegen, 1988). For positive affect, the internal consistency reliability was .83 among survivors, and .82 among caregivers. For negative affect, the internal consistency reliability was .87 among survivors, and .91 among caregivers. Perceived stress was measured with the Perceived Stress Scale (response options: $0=$ never, $4=$ very often; score range 0 to 40 with higher scores indicating more perceived stress)(Cohen, Kamarck, \& Mermelstein, 1983). The internal consistency reliability was .86 among survivors, and .90 among caregivers. Physical HRQOL. Fatigue was measured with the PROMIS short form 8a. The internal consistency reliability was .94 among survivors, and .93 among caregivers. Social HRQOL. Social isolation was measured with the PROMIS short form 8a. The internal consistency reliability was .98 among survivors, and .95 among caregivers. Emotional support was measured with the PROMIS 
short form $8 \mathrm{a}$. The internal consistency reliability was .95 among survivors, and .98 among caregivers. Informational support was measured with the PROMIS short form 8a. The internal consistency reliability was .96 among survivors and 1.0 among caregivers.

\section{Determination of feasibility and statistical analytic plan}

Feasibility to collect saliva to assess biomarkers was assessed according to the number of saliva samples collected from survivors and caregivers at appropriate time points according to the study protocol. Success was defined as obtaining biomarker data from $\geq 85 \%$ of samples per protocol. Saliva concentrations of cortisol were averaged across collection days in morning, afternoon, or evening because an effect of day was not expected. Likewise, concentrations of CRP in the morning were averaged across both collection days. For cortisol data, we also computed the slope of the change in cortisol from morning to afternoon to evening for each collection day (Saxbe, Repetti, \& Nishina, 2008), and then averaged cortisol slopes across both collection days to develop a metric of diurnal cortisol rhythm. We first examined biomarker and HRQOL variables by computing means and their standard errors by biomarker and time point (for cortisol only). Data that were not normally distributed (Shapiro-Wilk test) were natural log transformed before any inferential testing.

We then examined the association between biomarker variables (CRP, AM cortisol, PM cortisol, and cortisol slope) and HRQOL domains by computing partial and semipartial correlation coefficients controlling for BMI and chemotherapy treatment (survivors) and Pearson product-moment correlation coefficients (caregivers). We controlled for chemotherapy because evidence suggests that chemotherapy may be associated with fatigue by way of inflammatory mediators (Bower, 2019). In cases in which BMI data were missing for survivors $(n=4)$, we imputed BMI data over 10 separate data sets and reported the average of a given statistic from 
each of these 10 imputed datasets. Besides controlling for BMI and cancer treatments in survivors, we also controlled for stage of breast cancer based on prior research that different dimensions of HRQOL (e.g., depression) have been found to be related to stage of disease (Valderrama Rios \& Sánchez Pedraza, 2018). We did not calculate semipartial correlation coefficients controlling for BMI in caregivers because height and weight data were not available for over half of the caregivers in the study. A Spearman's rank correlation coefficient was computed instead for associations where one or both outcomes were not normally distributed. Correlations were classified as: very strong ( $\mathrm{r} \geq 0.8$ ), strong ( $\mathrm{r}$ range $0.6-0.79)$, moderate ( $\mathrm{r}$ range, 0.4-0.59), weak (r range, 0.20-0.39), and very weak (range: 0-0.19). Because the analytic focus of this pilot was not on statistical significance but on estimating the correlations for the relationship between biomarkers and HRQOL domains, we did not control for multiple testing.

\section{RESULTS}

\section{Sample description and feasibility to collect saliva for stress-related biomarkers}

About half of the survivors were receiving chemotherapy treatment, and a third were employed. Most survivors were married, had an annual income under $\$ 30,000$, and had a high school or middle school education. More caregivers (45\%) were employed, and about half of them earned $\$ 30,000$ or more per year and had at least some college. The majority of caregivers were married. Most caregivers were either spouses $(41 \%)$ or daughters $(27 \%)$. Survivors and caregivers on average were obese. Demographic characteristics of survivors and caregivers are shown in Table 1. Additionally, $45 \%$ of survivors and $36 \%$ of caregivers endorsed Spanish as their preferred language. All participants completed telephone HRQOL assessments, and $96 \%$ and $92 \%$ of saliva samples were collected from survivors and caregivers, respectively, and returned to the lab for analyses of concentrations of cortisol and CRP. Average cortisol slope was not computed for one survivor and two caregivers because 2 or more saliva samples were 
missing from samples collected from these individuals. Average concentrations of AM cortisol, PM cortisol, and CRP as well as cortisol slope are shown in Table 1.

\section{Associations between stress-related biomarkers and health-related quality of life domains}

We examined associations between cortisol variables (AM cortisol, PM cortisol, and average cortisol slope) and CRP and HRQOL outcomes in survivors (Table 2). Correlations or partial correlations ranged from strong to very weak across saliva biomarkers and HRQOL domains, with the strongest association found between PM saliva cortisol and fatigue (physical domain $)\left(r_{p}=0.63\right)$. In survivors we also noted moderate associations between fatigue and cortisol slope, and between PM saliva cortisol and perceived stress (psychological domain). There were also several weak correlations between saliva biomarkers and various measures across HRQOL domains (e.g., between AM saliva cortisol and depressive symptoms [PROMIS] in the psychological domain, and emotional support informational support in the social domain; between saliva CRP and fatigue in the physical domain, and positive affect and negative affect in the psychological domain) in survivors. We noted no strong associations between saliva biomarkers and HRQOL outcomes in caregivers (Table 3). However, we did find several moderate correlations between saliva CRP and HRQOL outcomes (e.g., emotional support, informational support). There were also several weak associations in caregivers (e.g., PM saliva cortisol and depressive symptoms [both PROMIS and CES-D]).

\section{DISCUSSION}

In this study we explored feasibility of collecting and analyzing saliva samples for diurnal cortisol rhythm and saliva CRP concentrations in a sample of Latina breast cancer survivors and their informal caregivers living in southern Arizona. Our sample of survivors and caregivers was comparable to the parent study [author citation] as well as our earlier study with Latina breast cancer survivors and their caregivers [author citation] in terms of age, 
employment status, income, and marital status. We found that collection of saliva for analyses of diurnal cortisol rhythm and CRP was feasible with the approach used, which included use of an instructions sheet as well as telephone and text reminders in the participant's preferred language. With respect to the relationship between biomarker variables and HRQOL, in survivors we observed a strong and statistically significant association between saliva concentrations of cortisol in saliva collected in the evening and fatigue, a key aspect of physical HRQOL. We also noted a trend association between cortisol slope and fatigue in survivors. Although there was a strong association between emotional support and saliva concentrations of CRP in caregivers, this was opposite of what we would have expected (i.e., higher CRP was associated with lower emotional support).

Although it may seem as though collection of saliva in the home setting for determination of stress-related biomarkers would be relatively straightforward, feasibility to collect saliva can vary depending on both study context and the population being studied. Indeed, there is evidence that Latinos may mistrust collection and storage of samples for biomarkers, have fears about being harmed through the collection of biomarkers, and worry about collection burden (G. Moreno et al., 2015). While most researchers conducting feasibility studies have reported success with saliva collection for biomarkers (including for diurnal cortisol rhythm), others have decided to not collect saliva to assess cortisol after encountering difficulties in feasibility studies (Halpern, Whitsel, Wagner, \& Harris, 2012). Our success with procedures to collect saliva in the home setting in this pilot study may be in part because of our use of an advisory board consisting of Latina survivors and their informal caregivers. The results presented here are therefore important, because they validate procedures used before in majority NHW samples of survivors and caregivers to collect saliva to assess cortisol and CRP for Latina breast cancer survivors and their 
informal caregivers. Our most notable finding therefore is that assessment of stress-related biomarkers in saliva can be acceptable to Latinas survivors and their caregivers. These procedures can be used in future research and practice to document the relationship between stress-related biomarkers and HRQOL in this group.

The observation of strong associations between fatigue and cortisol slope and fatigue and evening cortisol suggest that greater symptoms of fatigue in survivors may be associated with disruptions in the diurnal cortisol rhythm, especially at the end of the day. Evidence from prior research suggests that greater change in cortisol over the course of the day, and perhaps especially lower concentrations of cortisol in the evening, may be associated with improved physical HRQOL and other wellness domains in different populations (Stafford et al., 2017). Flatter cortisol slope has been associated with poorer mental and physical health in different populations, including in breast cancer survivors (Adam et al., 2017; Bower et al., 2005; Giese-Davis, Wilhelm, et al., 2006). Flatter cortisol slope has also been associated with reduced survival time in breast cancer survivors (Chida, Hamer, Wardle, \& Steptoe, 2008; Sephton, Sapolsky, Kraemer, \& Spiegel, 2000). Given that depression is also associated with reduced survival after breast cancer diagnosis (Falagas et al., 2007; Giese-Davis et al., 2011), it may be that alterations in stress-related biological pathways like cortisol are involved in causal links between HRQOL impairments like depression and reduced survival time (Spiegel \& GieseDavis, 2003). Although this is not a new hypothesis, to the best of our knowledge it has not been explicitly tested before in Latina breast cancer survivors or survivor-caregiver dyads. Strength of associations between psychological and social HRQOL assessments and diurnal cortisol rhythm outcomes (AM concentrations, PM concentrations, or slope) and saliva concentrations of CRP in survivors on the whole were not as robust. This may be due in part to the small sample size. It is also possible that different combinations of breast cancer 
therapies (i.e., chemotherapy alone or in combination with radiation or surgery), as well as participants being at different stages in treatment, may have weakened these associations in the sample as a whole (even though we controlled for these variables in our analyses).

Demographic characteristics (e.g., BMI) may have also reduced strength of associations between HRQOL and saliva biomarkers in the informal caregivers. We did find in caregivers that emotional support was moderately associated with saliva concentrations of CRP. Based on prior work on stress and measures of inflammation in a group of chronic caregivers (Miller et al., 2014), this result is opposite of what would be expected. However, caregivers in this study were mostly NHW, which limits generalizability of these findings (Cella et al., 2010). There is also evidence suggesting that individuals who seek out and find emotional support may experience high distress, including caregivers (Hou, Lau, Shum, Cheng, \& Lee, 2018). Distress accompanying emotional support may then drive production of inflammatory mediators, including CRP. Future studies should investigate this possibility with larger caregiver samples, including Latinos, as well as serial assessments of distress and emotional support.

Research with stress-related saliva biomarkers could one day facilitate more culturally congruent health care for Latina breast cancer survivors and their informal caregivers. These objective biomarkers provide important insights into biological pathways that are relevant for survivors and caregivers but that also can be activated by exposure to psychosocial stress. The biomarkers may also improve the interpretation of self-reported HRQOL in Latina survivors and their caregivers, especially when those HRQOL constructs may be influenced by stress exposure (e.g., depression, fatigue, perceived stress). Latinos in the United States may be at increased risk for poor health outcomes because the current sociopolitical climate could be a source of psychosocial stress (Gemmill et al., 2019; Hatzenbuehler et al., 2017), with resulting changes in the biological pathways. Cortisol and 
inflammatory mediators may therefore play an important role in HRQOL disparities experienced by Latina breast cancer survivors. Prior studies with ethnically mixed samples have found that HRQOL impairments experienced by breast cancer survivors (i.e., fatigue and depression) are associated with increased circulating concentrations of various inflammatory cytokines including interleukin (IL)-6, soluble tumor necrosis factor (TNF)receptor 2 (sTNF-R2), sIL-6 receptor, IL-1 receptor antagonist, and CRP (Bower, Ganz, Aziz, \& Fahey, 2002; Bower, Ganz, Aziz, Fahey, \& Cole, 2003; Bower et al., 2009; Collado-Hidalgo, Bower, Ganz, Cole, \& Irwin, 2006; Han et al., 2016; P. I. Moreno, Moskowitz, Ganz, \& Bower, 2016; Orre et al., 2011). Alterations in inflammatory markers in breast cancer survivors are likely more than epiphenomena, as inflammatory mediators have been shown to impact functioning of the brain in ways that encourage the development and severity of certain HRQOL impairments that are experienced by cancer survivors (Bower, Ganz, Irwin, Arevalo, \& Cole, 2011). Cortisol is essential for regulation of normal immune function, including inflammation, and insufficient cortisol or impaired tissue sensitivity to cortisol likely results in increased inflammation (Bower, 2014). In terms of how informal caregivers may be involved, others have begun to examine how diurnal cortisol rhythm may be interdependent in dementia patients and their caregivers (Hodgson \& Craemer, 2013). Thus, clinicians need to be aware of these negative consequences to biological pathways as part of quality care.

This pilot study has several notable limitations. First, the small sample size limited our ability to identify statistically meaningful associations between biomarkers and HRQOL measures in this population. Second, we relied on participants to indicate the time when they collected saliva samples, as opposed to using MEMS Caps or other electronic devices to record collection time. However, methods to electronically record times when containers holding saliva collection supplies (or medications) are opened have also been found to suffer from limitations 
(Ailinger, Black, \& Lima-Garcia, 2008; Hall et al., 2011). Third, the sample used in this pilot included survivors with different cancer treatment histories, and informal caregivers of varying ages and sex. Future studies with larger sample sizes will be able to better account for the possible contribution of these and other demographic characteristics in associations between biomarkers and different domains of HRQOL. Fourth, this pilot did not include NHW survivors and caregivers. Future research should include NHWs as well as survivors from other ethnic/ racial groups to understand how stress related to ethnic minority status may be involved. Finally, we were not able to collect height and weight information from all caregivers in this pilot study.

In summary, we demonstrated that collection of saliva from Latina breast cancer survivors and the informal caregivers to assess diurnal cortisol rhythm and CRP was feasible. We also report associations between these biomarkers and various dimensions of HRQOL for Latinas with breast cancer and their caregivers, which are new findings to the literature. Future studies should include biomarkers in research with Latina breast cancer survivors on how factors driving HRQOL disparities may include stress-related biological pathways, as well as the HRQOL and stress biology of informal caregivers. 


\section{REFERENCES}

Adam, E. K., Quinn, M. E., Tavernier, R., McQuillan, M. T., Dahlke, K. A., \& Gilbert, K. E. (2017). Diurnal cortisol slopes and mental and physical health outcomes: A systematic review and meta-analysis. Psychoneuroendocrinology, 83, 25-41.

Ailinger, R. L., Black, P. L., \& Lima-Garcia, N. (2008). Use of electronic monitoring in clinical nursing research. Clin Nurs Res, 17(2), 89-97.

Bower, J. E. (2014). Cancer-related fatigue--mechanisms, risk factors, and treatments. Nat Rev Clin Oncol, 11(10), 597-609.

Bower, J. E. (2019). The role of neuro-immune interactions in cancer-related fatigue: Biobehavioral risk factors and mechanisms. Cancer, 125(3), 353-364.

Bower, J. E., Ganz, P. A., Aziz, N., \& Fahey, J. L. (2002). Fatigue and proinflammatory cytokine activity in breast cancer survivors. Psychosomatic Medicine, 64(4), 604-611.

Bower, J. E., Ganz, P. A., Aziz, N., Fahey, J. L., \& Cole, S. W. (2003). T-cell homeostasis in breast cancer survivors with persistent fatigue. J Natl Cancer Inst, 95(15), 1165-1168.

Bower, J. E., Ganz, P. A., Dickerson, S. S., Petersen, L., Aziz, N., \& Fahey, J. L. (2005). Diurnal cortisol rhythm and fatigue in breast cancer survivors. Psychoneuroendocrinology, 30(1), 92-100.

Bower, J. E., Ganz, P. A., Irwin, M. R., Arevalo, J. M., \& Cole, S. W. (2011). Fatigue and gene expression in human leukocytes: increased NF-kappaB and decreased glucocorticoid 
signaling in breast cancer survivors with persistent fatigue. Brain Behav Immun, 25(1), 147-150.

Bower, J. E., Ganz, P. A., Tao, M. L., Hu, W., Belin, T. R., Sepah, S., . . Aziz, N. (2009). Inflammatory biomarkers and fatigue during radiation therapy for breast and prostate cancer. Clin Cancer Res, 15(17), 5534-5540.

Carver, C. S., Lehman, J. M., \& Antoni, M. H. (2003). Dispositional pessimism predicts illnessrelated disruption of social and recreational activities among breast cancer patients. $J$ Pers Soc Psychol, 84(4), 813-821.

Cella, D., Riley, W., Stone, A., Rothrock, N., Reeve, B., Yount, S., . . Hays, R. (2010). The Patient-Reported Outcomes Measurement Information System (PROMIS) developed and tested its first wave of adult self-reported health outcome item banks: 2005-2008. J Clin Epidemiol, 63(11), 1179-1194.

Chida, Y., Hamer, M., Wardle, J., \& Steptoe, A. (2008). Do stress-related psychosocial factors contribute to cancer incidence and survival? Nat Clin Pract Oncol, 5(8), 466-475.

Cohen, S., Kamarck, T., \& Mermelstein, R. (1983). A global measure of perceived stress. Journal of Health \& Social Behavior, 24(4), 385-396.

Collado-Hidalgo, A., Bower, J. E., Ganz, P. A., Cole, S. W., \& Irwin, M. R. (2006). Inflammatory biomarkers for persistent fatigue in breast cancer survivors. Clin Cancer Res, 12(9), 2759-2766. 
Cubukcu, M. (2018). Evaluation of quality of life in caregivers who are providing home care to cancer patients. Support Care Cancer, 26(5), 1457-1463.

Eversley, R., Estrin, D., Dibble, S., Wardlaw, L., Pedrosa, M., \& Favila-Penney, W. (2005). Post-treatment symptoms among ethnic minority breast cancer survivors. Oncol Nurs Forum, 32(2), 250-256.

Falagas, M. E., Zarkadoulia, E. A., Ioannidou, E. N., Peppas, G., Christodoulou, C., \& Rafailidis, P. I. (2007). The effect of psychosocial factors on breast cancer outcome: a systematic review. Breast Cancer Res, 9(4), R44.

Ferrell, B. R., Grant, M., Funk, B., Garcia, N., Otis-Green, S., \& Schaffner, M. L. (1996). Quality of life in breast cancer. Cancer Practice, 4(6), 331-340.

Gemmill, A., Catalano, R., Casey, J. A., Karasek, D., Alcala, H. E., Elser, H., \& Torres, J. M. (2019). Association of Preterm Births Among US Latina Women With the 2016 Presidential Election. JAMA Netw Open, 2(7), e197084.

Giese-Davis, J., Collie, K., Rancourt, K. M., Neri, E., Kraemer, H. C., \& Spiegel, D. (2011). Decrease in depression symptoms is associated with longer survival in patients with metastatic breast cancer: a secondary analysis. J Clin Oncol, 29(4), 413-420.

Giese-Davis, J., DiMiceli, S., Sephton, S., \& Spiegel, D. (2006). Emotional expression and diurnal cortisol slope in women with metastatic breast cancer in supportive-expressive group therapy: a preliminary study. Biol Psychol, 73(2), 190-198. 
Giese-Davis, J., Wilhelm, F. H., Conrad, A., Abercrombie, H. C., Sephton, S., Yutsis, M., .. . Spiegel, D. (2006). Depression and stress reactivity in metastatic breast cancer. Psychosomatic medicine, 68(5), 675-683.

Hall, D. L., Blyler, D., Allen, D., Mishel, M. H., Crandell, J., Germino, B. B., \& Porter, L. S. (2011). Predictors and patterns of participant adherence to a cortisol collection protocol. Psychoneuroendocrinology, 36(4), 540-546.

Halpern, C. T., Whitsel, E. A., Wagner, B., \& Harris, K. M. (2012). Challenges of measuring diurnal cortisol concentrations in a large population-based field study. Psychoneuroendocrinology, 37(4), 499-508.

Han, T. J., Felger, J. C., Lee, A., Mister, D., Miller, A. H., \& Torres, M. A. (2016). Association of childhood trauma with fatigue, depression, stress, and inflammation in breast cancer patients undergoing radiotherapy. Psychooncology, 25(2), 187-193.

Hatzenbuehler, M. L., Prins, S. J., Flake, M., Philbin, M., Frazer, M. S., Hagen, D., \& Hirsch, J. (2017). Immigration policies and mental health morbidity among Latinos: A state-level analysis. Soc Sci Med, 174, 169-178.

Ho, R. T., Fong, T. C., Chan, C. K., \& Chan, C. L. (2013). The associations between diurnal cortisol patterns, self-perceived social support, and sleep behavior in Chinese breast cancer patients. Psychoneuroendocrinology, 38(10), 2337-2342.

Hodgson, N., \& Craemer, G. (2013). In sickness and in health: Coregulation of spousal caregivers' and dementia patients' cortisol levels, relationship quality and health outcomes. Alzheimer's and Dementia, 9(4 Supplement), P481. 
Hou, W. K., Lau, K. M., Shum, T. C. Y., Cheng, A. C. K., \& Lee, T. M. C. (2018). Do concordances of social support and relationship quality predict psychological distress and well-being of cancer patients and caregivers? Eur J Cancer Care (Engl), 27(4), e12857.

Juarez Garcia, D. M., Landero Hernandez, R., González-Ramírez, M., \& Jaime, L. (2016).

Diurnal cortisol variatiojn and its relationship with stress, optimism, and coping strategies in women with breast cancer. Acta Colombiana de Psicologia, 19(1), 113-122.

Julious, S. A. (2005). Sample size of 12 per group rule of thumb for a pilot study. Pharmaceutical Statistics: The Journal of Applied Statistics in the Pharmaceutical Industry, 4(4), 287-291.

Kent, E. E., Rowland, J. H., Northouse, L., Litzelman, K., Chou, W. Y., Shelburne, N., .. . Huss, K. (2016). Caring for caregivers and patients: Research and clinical priorities for informal cancer caregiving. Cancer, 122(13), 1987-1995.

Kirschbaum, C., \& Hellhammer, D. H. (1994). Salivary cortisol in psychoneuroendocrine research: recent developments and applications. Psychoneuroendocrinology, 19(4), 313333.

Lambert, S. D., Girgis, A., Lecathelinais, C., \& Stacey, F. (2013). Walking a mile in their shoes: anxiety and depression among partners and caregivers of cancer survivors at 6 and 12 months post-diagnosis. Support Care Cancer, 21(1), 75-85.

Luckett, T., Goldstein, D., Butow, P. N., Gebski, V., Aldridge, L. J., McGrane, J., ... King, M. T. (2011). Psychological morbidity and quality of life of ethnic minority patients with cancer: a systematic review and meta-analysis. Lancet Oncol, 12(13), 1240-1248. 
Miller, G. E., Murphy, M. L., Cashman, R., Ma, R., Ma, J., Arevalo, J. M., . . Cole, S. W. (2014). Greater inflammatory activity and blunted glucocorticoid signaling in monocytes of chronically stressed caregivers. Brain Behav Immun, 41, 191-199.

Moreno, G., Mangione, C. M., Meza, C. E., Kwon, I., Seeman, T., Trejo, L., . . Sarkisian, C. A. (2015). Perceptions from latino and african american older adults about biological markers in research. Ethn Dis, 25(3), 355-362.

Moreno, P. I., Moskowitz, A. L., Ganz, P. A., \& Bower, J. E. (2016). Positive Affect and Inflammatory Activity in Breast Cancer Survivors: Examining the Role of Affective Arousal. Psychosom Med, 78(5), 532-541.

Munoz, J., Rush, C., Flashner, B., Cibrian, G., Martinez, S., Campos, C., . . . Graves, K. D. (2017). "The song inside": "La canción por dentro"-Individual and dyadic impact of breast cancer for caregivers of Latina survivors. Psychooncology, 26(10), 1700-1703.

Orre, I. J., Reinertsen, K. V., Aukrust, P., Dahl, A. A., Fossa, S. D., Ueland, T., \& Murison, R. (2011). Higher levels of fatigue are associated with higher CRP levels in disease-free breast cancer survivors. J Psychosom Res, 71(3), 136-141.

Ouellet-Morin, I., Danese, A., Williams, B., \& Arseneault, L. (2011). Validation of a highsensitivity assay for C-reactive protein in human saliva. Brain Behav Immun, 25(4), 640646.

Park, J., Ross, A., Klagholz, S. D., \& Bevans, M. F. (2018). The Role of Biomarkers in Research on Caregivers for Cancer Patients: A Scoping Review. Biol Res Nurs, 20(3), 300-311. 
Radloff, S. L. (1977). The CES-D Scale: A Self-Report Depression Scale for Research in the General Population. Applied Psychological Measurement 1(3), 385-401.

Saxbe, D. E., Repetti, R. L., \& Nishina, A. (2008). Marital satisfaction, recovery from work, and diurnal cortisol among men and women. Health Psychol, 27(1), 15-25.

Segrin, C., \& Badger, T. A. (2014). Psychological and physical distress are interdependent in breast cancer survivors and their partners. Psychol Health Med, 19(6), 716-723.

Sephton, S. E., Dhabhar, F. S., Keuroghlian, A. S., Giese-Davis, J., McEwen, B. S., Ionan, A. C., \& Spiegel, D. (2009). Depression, cortisol, and suppressed cell-mediated immunity in metastatic breast cancer. Brain Behav Immun, 23(8), 1148-1155.

Sephton, S. E., Sapolsky, R. M., Kraemer, H. C., \& Spiegel, D. (2000). Diurnal cortisol rhythm as a predictor of breast cancer survival.[see comment]. Journal of the National Cancer Institute, 92(12), 994-1000.

Spencer, S. M., Lehman, J. M., Wynings, C., Arena, P., Carver, C. S., Antoni, M. H., .. . Love, N. (1999). Concerns about breast cancer and relations to psychosocial well-being in a multiethnic sample of early-stage patients. Health Psychol, 18(2), 159-168.

Spiegel, D., \& Giese-Davis, J. (2003). Depression and cancer: mechanisms and disease progression. Biol Psychiatry, 54(3), 269-282.

Spiegel, D., Giese-Davis, J., Taylor, C.B., \& Kraemer, H. (2006). Stress sensitivity in metastatic breast cancer: Analyisis of the hypothalmic-pituitary-adrenal axis function. Psychoneuroendocrinology, 31, 1231-1244. 
Stafford, M., Ben-Shlomo, Y., Cooper, C., Gale, C., Gardner, M. P., Geoffroy, M. C., ... Cooper, R. (2017). Diurnal cortisol and mental well-being in middle and older age: evidence from four cohort studies. BMJ Open, 7(10), e016085.

Turner-Cobb, J. M., Sephton, S. E., Koopman, C., Blake-Mortimer, J., \& Spiegel, D. (2000). Social support and salivary cortisol in women with metastatic breast cancer. Psychosom Med, 62(3), 337-345.

Valderrama Rios, M. C., \& Sánchez Pedraza, R. (2018). Trastornos de ansiedad y depresión en relación con la calidad de vida de pacientes con cáncer de mama en estadio localmente avanzado o diseminado (Anxiety and depression disorders in relation to the quality of life of breast cancer patients with locally advanced or disseminated stage). Revista Colombiana de Psiquiatría, 47(4), 211-220.

Watson, D., Clark, L. A., \& Tellegen, A. (1988). Development and validation of brief measures of positive and negative affect: the PANAS scales. J Pers Soc Psychol, 54(6), 1063-1070.

Xiao, C., Miller, A. H., Felger, J., Mister, D., Liu, T., \& Torres, M. A. (2017). Depressive symptoms and inflammation are independent risk factors of fatigue in breast cancer survivors. Psychol Med, 47(10), 1733-1743.

Yanez, B., McGinty, H. L., Buitrago, D., Ramirez, A. G., \& Penedo, F. J. (2016). Cancer Outcomes in Hispanics/Latinos in the United States: An Integrative Review and Conceptual Model of Determinants of Health. Journal of Latioa/o Psychology, 4(2), 114129. 
Yanez, B., Thompson, E. H., \& Stanton, A. L. (2011). Quality of life among Latina breast cancer patients: a systematic review of the literature. J Cancer Surviv, 5(2), 191-207. 
Table 1. Breast cancer survivor and caregiver demographic characteristics, survivor treatment information, average survivor and caregiver biomarker concentrations, and average survivor and caregiver cortisol slope.

Age, mean (SD)

Female

Body mass index (SD)

Breast Cancer Stage***

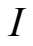

II

III

IV

Current cancer treatment

chemotherapy

radiation

surgery

Completed cancer treatment

chemotherapy (\%)

radiation $(\%)$

surgery $(\%)$

Employment

Unemployed, seeking

Employed

Retired, disabled, other

Income

Did not answer

Under $\$ 10,000$

$\$ 10,000-\$ 29,999$

$\$ 30,000-\$ 59,999$

$\$ 60,000$ or more

Highest Level of Education

Elementary

Middle school

High school

At least some college

Relationship to survivor

Spouse

Daughter

Mother

Son

Friend

Sibling

\section{Marital status}

Married/partnered

Not married/ partnered
Survivor $(\mathrm{n}=22)$

$52.41(11.25)$

$22(100 \%)$

$32.38(7.00) *$

$4(18 \%)$

$6(27 \%)$

$6(27 \%)$

$3(14 \%)$

$13(59.1 \%)$

$3(13.6 \%)$

$2(9.1 \%)$

5 (22.7)

3 (13.6)

$11(50)$

$4(18 \%)$

$7(32 \%)$

$11(50 \%)$

$0(0 \%)$

$8(36 \%)$

$7(32 \%)$

$3(14 \%)$

$4(18 \%)$

$1(5 \%)$

$5(23 \%)$

$5(25 \%)$

$6(27 \%)$

$13(59 \%)$

$9(41 \%)$
Caregiver $(\mathrm{n}=22)$

$45.32(14.77)$

$12(54.5 \%)$

$30.07(7.00)^{* *}$

\section{Saliva cortisol}

$5(23 \%)$

$10(45 \%)$

$7(32 \%)$

$1(5 \%)$

$3(14 \%)$

$7(32 \%)$

$5(23 \%)$

$6(27 \%)$

$1(5 \%)$

$2(9 \%)$

7 (32\%)

$12(55 \%)$

$9(41 \%)$

$6(27 \%)$

$2(9 \%)$

$2(9 \%)$

$2(9 \%)$

$1(5 \%)$

$16(73 \%)$

$6(27 \%)$ 
Table 2: Partial correlations between health-related quality of life (HRQOL) domains and saliva biomarkers, controlling for body mass index and chemotherapy (current and past), in Latina breast cancer survivors.

\section{Biomarker}

\begin{tabular}{|c|c|c|c|c|}
\hline $\begin{array}{c}\text { HRQOL } \\
\text { Dimension }\end{array}$ & saliva CRP & $\begin{array}{c}\text { morning saliva } \\
\text { cortisol }\end{array}$ & $\begin{array}{c}\text { evening saliva } \\
\text { cortisol }\end{array}$ & $\begin{array}{c}\text { saliva cortisol } \\
\text { slope }\end{array}$ \\
\hline \multicolumn{5}{|l|}{ Psychological } \\
\hline $\begin{array}{l}\text { depression } \\
\text { (PROMIS) }\end{array}$ & 0.218 & 0.201 & -0.210 & 0.020 \\
\hline $\begin{array}{c}\text { depression } \\
\text { (CES-D) }\end{array}$ & -0.003 & -0.089 & -0.139 & -0.057 \\
\hline anxiety & 0.234 & 0.136 & -0.273 & -0.054 \\
\hline positive affect & -0.238 & -0.088 & 0.306 & 0.126 \\
\hline $\begin{array}{l}\text { negative } \\
\text { affect }\end{array}$ & 0.255 & 0.123 & 0.013 & -0.094 \\
\hline $\begin{array}{l}\text { perceived } \\
\text { stress }\end{array}$ & 0.095 & 0.068 & -0.416 & -0.125 \\
\hline$\frac{\text { Physical }}{\text { fatigue }^{\S}}$ & \multicolumn{3}{|c|}{ Physical } & $0.494^{\dagger}$ \\
\hline \multicolumn{5}{|l|}{$\underline{\text { Social }}$} \\
\hline $\begin{array}{c}\text { social } \\
\text { isolation }\end{array}$ & -0.173 & -0.057 & -0.150 & -0.010 \\
\hline $\begin{array}{l}\text { emotional } \\
\text { support }\end{array}$ & 0.280 & 0.347 & -0.001 & 0.047 \\
\hline $\begin{array}{l}\text { informational } \\
\text { support }\end{array}$ & 0.346 & 0.385 & -0.058 & -0.079 \\
\hline
\end{tabular}

$\S_{\text {only } 14}$ cases available with this outcome $\dagger \mathrm{p}=0.088, \uparrow \dagger \mathrm{p}<0.01$ 
Table 3: Pearson correlation coefficients (unless otherwise noted) between health-related quality of life (HRQOL) domains and saliva biomarkers in informal caregivers of Latina breast cancer survivors.

\section{Biomarker}

\begin{tabular}{|c|c|c|c|c|}
\hline $\begin{array}{c}\text { HRQOL } \\
\text { Dimension }\end{array}$ & saliva CRP* & $\begin{array}{l}\text { morning saliva } \\
\text { cortisol }\end{array}$ & $\begin{array}{l}\text { evening saliva } \\
\text { cortisol }\end{array}$ & $\begin{array}{l}\text { saliva cortisol } \\
\text { slope* }\end{array}$ \\
\hline \multicolumn{5}{|l|}{ Psychological } \\
\hline $\begin{array}{l}\text { depression } \\
\text { (PROMIS) }\end{array}$ & -0.202 & -0.002 & 0.254 & 0.198 \\
\hline $\begin{array}{c}\text { depression } \\
\text { (CES-D) }\end{array}$ & -0.034 & -0.034 & 0.223 & 0.223 \\
\hline anxiety & $-0.458^{\dagger}$ & 0.214 & 0.147 & 0.047 \\
\hline positive affect & -0.137 & 0.392 & -0.042 & -0.206 \\
\hline $\begin{array}{c}\text { negative } \\
\text { affect }\end{array}$ & -0.354 & -0.047 & 0.044 & 0.180 \\
\hline $\begin{array}{l}\text { perceived } \\
\text { stress }\end{array}$ & -0.214 & -0.064 & -0.021 & -0.007 \\
\hline$\frac{\text { Physical }}{\text { fatigue }^{\S}}$ & 0.216 & -0.170 & 0.407 & 0.200 \\
\hline$\underline{\text { Social }}$ & & & & \\
\hline $\begin{array}{c}\text { social } \\
\text { isolation }\end{array}$ & 0.110 & 0.233 & 0.142 & -0.031 \\
\hline $\begin{array}{l}\text { emotional } \\
\text { support }\end{array}$ & $0.500^{\dagger}$ & 0.050 & -0.044 & -0.235 \\
\hline $\begin{array}{l}\text { informational } \\
\text { support }\end{array}$ & 0.414 & -0.082 & -0.005 & 0.016 \\
\hline
\end{tabular}

* Spearman's rank correlation coefficients ${ }^{\S}$ only 14 cases available with this outcome ${ }^{\dagger} \mathrm{p}<0.05$ 


$\begin{array}{cll}\text { AM, mean }(S D) & 0.29 \mu \mathrm{g} / \mathrm{dL}(0.16) & 0.41 \mu \mathrm{g} / \mathrm{dL}(0.52) \\ P M, \text { mean }(S D) & 0.25 \mu \mathrm{g} / \mathrm{dL}(0.54) & 0.10 \mu \mathrm{g} / \mathrm{dL}(0.06) \\ \text { Slope } & -0.036 & -0.551 \\ \text { Saliva C-reactive protein (SD) } & 16,103.82(17,900.02) & 11,469.07 \mathrm{pg} / \mathrm{mL}(16,764.69) \\ * \mathrm{n}=18 ; * * \mathrm{n}=10, * * * \mathrm{n}=19 & & \end{array}$

